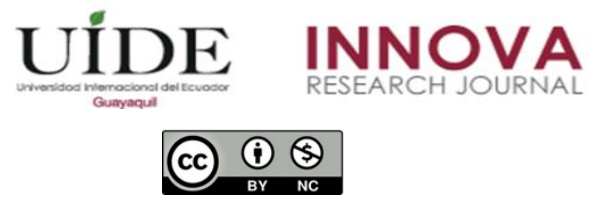

INNOVA Research Journal, ISSN 2477-9024

(Septiembre-Diciembre 2019). Vol. 4, No.3.1 pp. 18-29

DOI: https://doi.org/10.33890/innova.v4.n3.1.2019.1064

URL: http://revistas.uide.edu.ec/index.php/innova/index

Correo: innova@uide.edu.ec

\title{
Determinantes de la Carga Fiscal: Caso de las empresas ecuatorianas
}

\section{Determinants of the Tax Burden: The Case of Ecuadorian companies}

Karen Michel Serrano Orellana

Universidad Metropolitana, Ecuador

María Cristina Chiriboga Ayala

Universidad de Guayaquil, Ecuador

Autor para correspondencia: kserrano@umet.edu.ec; mchiriboga@ug.edu.ec

Fecha de recepción: 12 de julio de 2019 - Fecha de aceptación: 29 de octubre de 2019

\section{Resumen}

Estudios previos realizados en Estados Unidos, Europa y Asia han demostrado que los factores empresariales inciden en la presión fiscal que soportan las empresas, por lo cual, en la presente investigación se intenta dar luces de los determinantes de la carga fiscal de las empresas ecuatorianas, seleccionando una muestra de 27.877 empresas en el periodo 2014-2017. Dentro de los factores analizados se seleccionaron: el tamaño de la empresa, el nivel de apalancamiento, las decisiones de inversión, la rentabilidad económica, las operaciones en el extranjero y como proxi de la carga fiscal se tomó a la tasa impositiva efectiva (TIE). Empleando datos de panel se encontró que la tasa impositiva efectiva tiene una relación negativa y significativa con las variables tamaño y rentabilidad, es decir, que las empresas más grandes y más rentables soportan menor presión fiscal. En contraparte, se evidencia una relación positiva con la variable apalancamiento, lo que significa que las empresas más endeudadas soportan una mayor carga fiscal. Las variables intensidad del capital, intensidad del inventario y operaciones en el exterior no son significativas y por ende no son determinantes de la carga fiscal de las empresas ecuatorianas.

Palabras Claves: tasa impositiva efectiva; presión fiscal; factores empresariales; tamaño; apalancamiento

Abstract: Previous studies carried out in the United States, Europe and Asia have shown that business factors influence the tax burden borne by companies, which is why, in this study, we analyzed the determinants of the tax burden of Ecuadorian companies, for which a sample of 27877 firms were selected in the 2014-2017 period. Among the factors analyzed were selected: the size of the company, the leverage, the investment decisions, the profitability and operations abroad and as a proxy of the tax burden it was selected the effective tax rate (TIE). Using panel data, it was found that the effective tax rate has a negative and significant relationship with the variables size and profitability, that is, that larger and more profitable companies bear lower tax pressure. On the other hand, there is evidence of a positive relationship with the variable leverage, which means that the most indebted companies bear a greater fiscal burden. The variables capital intensity, inventory intensity and operations abroad are not significant, so it can be concluded that they are not determinants of the tax burden.

Key words: effective tax rate; tax burden; business factors; size; leverage 


\section{Introducción}

La carga fiscal que soportan las empresas y sus factores determinantes, son de gran importancia para los ejecutivos y analistas que desean conocer cómo inciden las decisiones de inversión y financiación en la aplicación del impuesto sobre sociedades. La evolución de los sistemas fiscales ha atraído en las últimas décadas una creciente atención en la investigación. A pesar de las tendencias generales en tributación, el estudio de las tasas de impuesto efectivas y sus determinantes han llamado la atención hacia una intensa investigación por parte de países como Estados Unidos, China o la Unión Europea.

Un gran número de estudios utilizan como proxi de la carga fiscal a la tasa impositiva efectiva (TIE), la cual brinda información más allá de las tasas fiscales reglamentarias, debido a que revelan las características propias de la real distribución del sistema impositivo. En Ecuador, el cálculo del impuesto a sociedades (IS) o impuesto a la renta (IR) se realiza sobre la base imponible de ingresos gravados menos todos los costos y gastos imputables a tales ingresos.

El estudio de Fernández, García y Martínez (2019) encuentra que las variables tamaño, apalancamiento, investigación y desarrollo, rentabilidad entre otras tienen mayor relevancia en determinar la carga fiscal en las empresas privadas que en las de propiedad estatal, dado que en las primeras estos factores son significativos, manifestando adicionalmente que esto puede suceder ya que las compañías privadas asignan recursos para aplicar estrategias de elusión fiscal.

En Ecuador, hasta donde se conoce no se han realizado investigaciones basadas en la tasa impositiva efectiva, por lo cual el objetivo de la presente investigación es determinar si los distintos factores empresariales, tales como el tamaño, la rentabilidad económica, apalancamiento, operaciones con el exterior e intensidad de las inversiones, afectan la presión fiscal de las empresas ecuatorianas.

El presente estudio comprende dos etapas: en la primera, se seleccionaron un total de 27877 empresas ecuatorianas que presentan sus estados financieros a la Superintendencia de Compañías, Valores y Seguros para poder extraer las principales cuentas que permitieron conocer la situación económica, financiera y fiscal en el periodo 2014-2017, además se procedió a calcular algunas métricas de interés para el análisis como la rentabilidad económica y la tasa efectiva impositiva. Y en la segunda etapa, se analizaron los determinantes de la carga fiscal en las empresas ecuatorianas y su incidencia en la tasa impositiva efectiva.

Los resultados obtenidos muestran que el tamaño, el apalancamiento y la rentabilidad económica afectan la carga impositiva de las empresas ecuatorianas, mientras que las decisiones de inversión y las operaciones con el extranjero no inciden de manera significativa en la presión fiscal que soportan las empresas.

Finalmente, el estudio es relevante debido a la carencia de este tipo de investigaciones en Ecuador. Este nuevo conocimiento puede contribuir a que las empresas conozcan cómo los diversos factores empresariales pueden afectar de manera positiva o negativa a la presión fiscal que soportan en sus actividades. La investigación se estructura de la siguiente manera: en la Sección 2 hace una breve reseña de literatura previa, describe diferentes teorías y establece las Esta obra se comparte bajo la licencia Creative Common Atribución-No Comercial 4.0 International (CC BY-NC 4.0) Revista de la Universidad Internacional del Ecuador. URL: https://www.uide.edu.ec/ 
hipótesis; la Sección 3 describe la muestra seleccionada y la base de datos utilizada; la Sección 4 describe el modelo econométrico y explica las variables utilizadas en el estudio; la Sección 5 explica los resultados empíricos; y la Sección 6 detalla las principales conclusiones.

\section{Revisión de la literatura}

La literatura empírica enfocada en estudiar el papel de las tasas impositivas efectivas ha crecido en los últimos años. Uno de los primeros trabajos de investigación que muestra la relación existente entre el tamaño de las empresas y la tasa impositiva efectiva, es el realizado por Zimmerman (1983) cuyos resultados muestran que las compañías más grandes tienen mayor (TIE) que las empresas más pequeñas.

Respecto al tamaño de la empresa existen dos teorías o puntos de vista: por un lado, la teoría del poder político que se centra en que las empresas de mayor tamaño se involucran agresivamente en la planificación fiscal y utilizan su influencia para promover políticas fiscales que le favorezcan con un mayor ahorro (Becker, 1983). Por otro lado, la teoría del costo político se fundamenta en que las empresas de mayor tamaño, tienen mayor visibilidad y éxito, por ende, es más probable que sean objeto de presiones fiscales que la afecten en mayor medida (Watts y Zimmerman, 1986).

Delgado, Fernández y Martínez (2012), analizan los factores determinantes de la tasa impositiva efectiva en empresas de Estados Unidos y encuentran que existe una relación no lineal entre la (TIE) y el tamaño, es decir que solamente las empresas que sobrepasen un especifico tamaño pueden reducir la presión fiscal que soportan.

Desde otro punto de vista, Irlacher y Unger (2018) intentan dar una explicación a la relación negativa entre las (TIE) y el tamaño de la empresa incluso sin transferencia de ganancias o el uso de paraísos fiscales, concluyendo que una transferencia imperfecta de los impuestos corporativos a los precios y a la deducción parcial de los costos de producción, especialmente para las empresas que tiene mayor producción, reducen los beneficios antes de impuestos.

Posteriormente, la investigación realizada por Delgado, Fernández y Martínez (2018) intenta analizar la relación entre la tasa impositiva efectiva y el tamaño, con la finalidad de comprobar si prevalece la teoría del poder político o del costo político en las empresas alemanas. Los resultados de la investigación son interesantes, debido a que al utilizar una regresión cuantílica se obtiene que inicialmente sobresale la teoría del costo político, pero a partir del cuantil 0.5 prevalece la teoría del poder político.

Adhikari, Derashid y Zhang (2006), analizan la relación entre las conexiones políticas y las tasas impositivas efectivas, y encuentran que las empresas que poseen conexiones políticas tienen una menor presión fiscal en comparación a las otras, por lo cual consideran que este es un determinante esencial.

Existe otras investigaciones como la de Stickney y McGee (1982) en la cual además del tamaño consideraron otros determinantes de la (TIE), como la intensidad en capital, el volumen de deuda, la inversión en recursos naturales y la medida de sus operaciones en el exterior, Esta obra se comparte bajo la licencia Creative Common Atribución-No Comercial 4.0 International (CC BY-NC 4.0) Revista de la Universidad Internacional del Ecuador. URL: https://www.uide.edu.ec/ 
concluyendo que existe una relación inversa entre las tres primeras y la tasa impositiva efectiva, mientras que el tamaño y las operaciones en el exterior no son relevantes en la presión fiscal que soportan. Por otro lado, Richardson y Lanis (2007) muestran que el tamaño, el nivel de endeudamiento y la mezcla de los activos, afectan significativamente a la tasa impositiva de las empresas australianas.

Fonseca, Fernández y Martínez (2019) analizan los efectos de los factores empresariales y las variables institucionales de 63 países en la presión fiscal que soportan las empresas cotizadas, donde muestran que el volumen del inventario y la rentabilidad tienen una relación positiva con la (TIE), mientras que el nivel de apalancamiento, la intensidad en capital tienen una asociación negativa con la presión fiscal, e indican que estos factores pueden verse condicionados de acuerdo al país donde se encuentren ubicada la empresa.

Lazar (2014) analiza los determinantes de las tasas impositivas efectivas en empresas cotizadas en Rumanía, los resultados obtenidos muestran que el apalancamiento, la intensidad del capital y la acumulación de pérdidas tienen una relación negativa con la (TIE), mientras que la rentabilidad tiene una asociación positiva. Por otro lado, el estudio encuentra que ni el tamaño de la empresa, ni la intensidad en mano de obra, tienen efecto en la presión fiscal, por lo cual indican que no existe evidencia que las empresas rumanas de mayor tamaño se involucren en actividades de planificación fiscal más fuertes.

Vintila, Gherghina y Paunescu, (2018), al analizar los factores determinantes de la carga fiscal en empresas cotizadas de Rumania, Hungría, Polonia, Bulgaria y Eslovenia encuentran que existe una relación positiva entre la tasa impositiva efectiva y la rentabilidad, la deuda, la intensidad de capital e inventario y el tamaño de la empresa.

Así mismo el estudio de Fernández (2004), encuentra que las empresas españolas que poseen mayor endeudamiento soportan menor presión fiscal mientras que las más rentables soportan mayor presión. De igual forma la investigación de Chiou, Hsieh, y Lin (2012) encuentra que, bajo diferentes mediciones de la tasa impositiva efectiva, la rentabilidad económica es siempre positiva y significativa, es decir que las empresas chinas más rentables tienen mayor carga fiscal.

Molina y Barberá (2017) muestran que en las empresas de la zona euro, la intensidad en capital y la rentabilidad económica tienen una relación negativa con la presión fiscal mientras que existe una relación positiva con el nivel de endeudamiento y la rentabilidad financiera. Respecto al tamaño encuentran que existe una relación no lineal, es decir las empresas grandes soportan una carga fiscal mayor, pero a partir de un tamaño la relación se invierte y tienen una menor carga mientras más grandes son.

Al igual que las investigaciones indicadas anteriormente, existen otros estudios empíricos previos donde se analizan los determinantes específicos de las (TIE), realizados en Estados Unidos, China, Australia, la Unión Europea, cuyos resultados difieren en cuanto a la relación positiva o negativa, así como al nivel de significancia. Mientras que entre los métodos de estimación utilizados en la literatura se han encontrado: el método de mínimos cuadrados 
ordinarios agrupados, método de efectos fijos, método de efectos aleatorios y regresión por cuantiles.

Si bien es cierto los resultados son interesantes respecto a cada país, no se puede indicar que los resultados son extrapolables entre países debido a las características propias e inherentes a cada jurisdicción.

\section{Planteamiento de Hipótesis}

Como se detalló en el apartado anterior, las investigaciones previas realizadas en Estados Unidos y diversos países europeos muestran que gran parte de las decisiones empresariales afectan a la carga fiscal que estas soportan. En Ecuador no se han realizado estudios similares, de forma que es importante determinar si las variables estudiadas en investigaciones previas tienen impacto en la presión fiscal de las empresas ecuatorianas y de tenerlas cual es la relación existente entre ellas. Como proxi de la presión fiscal se toma a la tasa impositiva efectiva (TIE).

Para determinar el impacto se han planteado en este estudio cinco hipótesis, las cuales permitirán contrastar el efecto de la información contable en la tasa impositiva efectiva.

H1: El tamaño de la empresa afecta la carga fiscal de las compañías ecuatorianas.

H2: El nivel de apalancamiento de una empresa afecta la carga fiscal de las compañías ecuatorianas.

H3: Las decisiones de inversión afectan la carga fiscal de las empresas ecuatorianas.

H4: La rentabilidad económica afecta la carga fiscal de las empresas ecuatorianas.

H5: Las operaciones extranjeras de las empresas afectan la carga fiscal de las compañías ecuatorianas.

\section{Muestra}

Para la presente investigación se utilizó la base de datos de la Superintendencia de Compañías, Valores y Seguros del Ecuador, la cual contiene la información financiera de las empresas ecuatorianas. Este artículo utiliza información anual de los estados financieras en el periodo 2014-2017.

Se tomó como parte de este estudio a las empresas pertenecientes a los sectores de actividades de alojamiento y de servicio de comidas, actividades inmobiliarias, agricultura, ganadería, silvicultura y pesca, construcción, explotación de minas y canteras, industrias manufactureras, información y comunicación, transporte y almacenamiento, suministro de electricidad, gas, y vapor; fueron excluidas del análisis las pertenecientes al sector financiero debido a las características especiales de su actividad.

Inicialmente se tomaron como referencia a las empresas activas en Ecuador que están obligadas a llevar contabilidad dando un total de 73475 empresas, se eliminaron aquellas que poseían un total de activos menor a 50000 dólares americanos quedando 28504 empresas con un total de 77681 observaciones. Posteriormente se eliminaron las observaciones con tasas impositivas efectivas superiores a uno, como referencia de metodologías usadas en estudios Esta obra se comparte bajo la licencia Creative Common Atribución-No Comercial 4.0 International (CC BY-NC 4.0) Revista de la Universidad Internacional del Ecuador. URL: https://www.uide.edu.ec/ 
previos. Finalmente, la muestra seleccionada comprende 27877 empresas y 75289 observaciones.

\section{Presentación del modelo y descripción de las variables.}

Para la presente investigación se utilizó una regresión de datos de panel, en la cual la variable dependiente es la tasa impositiva efectiva $\left(T I E_{i t}\right)$. Para obtener esta variable se procedió a dividir el impuesto causado para los beneficios antes de impuestos y repartición de utilidades de la empresa i en el año t. Para esta medida se eliminaron las observaciones con impuesto causado negativo y también las observaciones que contenían valores superiores a uno, para que la variable se encuentre entre cero y uno. La ecuación utilizada se explica a continuación:

$$
\begin{aligned}
\text { TIE }_{i t}=\beta_{0}+ & \beta_{1} \text { Tamaño }_{i t}+\beta_{2} \text { Apalancamiento }_{i t}+\beta_{3} \text { IntensidadCapital }_{i t} \\
& +\beta_{4} \text { IntensidadInventario }_{i t}+\beta_{5} \text { ROA }_{i t}+\beta_{6} \text { Activ. Extran }_{i t} \\
& + \text { YearDummies }+\varepsilon_{i t}
\end{aligned}
$$

Donde,

- Tamañoit: se refiere al tamaño de la empresa i en el año t, se calcula como el logaritmo natural de total de activos de cada empresa de la muestra.

- Apalancamientoit: se refiere al apalancamiento, se calcula como la ratio de deuda total sobre el total de activos, de la empresa i en el año t.

- IntensidadCapital ${ }_{i t}$ : se refiere a la intensidad en capital, se calcula como la ratio de activos fijos sobre activos totales, de la empresa i en el año t.

- IntensidadInventario it: se refiere a la intensidad en inventarios, se calcula como la ratio de inventarios sobre el total de activos de la empresa i en el año t.

- ROA it: es la rentabilidad económica que se calcula como la relación entre el beneficio antes de impuesto sobre los activos totales, de la empresa i en el año t.

- Activ.Extran it: se refiere a la actividad en el extranjero, la cual es una variable dummy que toma el valor de 1 si tiene operaciones en el extranjero y cero caso contrario.

- YearDummies: Variable dummy de año para controlar los efectos del tiempo.

Para determinar si los efectos individuales se encuentran relacionados con las variables explicativas, se aplicó el test de Hausman, que permite la comparación de las estimaciones del modelo de efectos fijos con efectos aleatorios. Una vez realizada la prueba se encontraron diferencias sistemáticas, pues se obtuvo $\chi^{2}=434.43$ con una Prob $>\chi^{2}=0.000$, por lo cual se rechaza la hipótesis nula; sugiriendo la utilización del modelo de efectos fijos.

\section{Resultados}

\section{Análisis de Estadísticas Descriptivas}

En la tabla 1 se presentan las estadísticas descriptivas de las variables utilizadas. En el panel A se muestra la media, mediana, cuartiles, los valores mínimos y máximos mientras que en el Panel B se puede observar la matriz de correlación de Pearson. 
De los principales resultados que se muestran en el Panel A se puede indicar que la media de las tasas efectivas de las empresas ecuatorianas en los 4 años de estudio es de 28,67\% y la mediana de $24,11 \%$, superiores a la tasa legal establecida en Ecuador en este periodo, la cual es del 22\%. Es importante mencionar que la menor tasa efectiva de las empresas es del $0 \%$ y la mayor del 99,9\%, dando como primer análisis que las tasas efectivas difieren entre las empresas y en el tiempo. Se puede evidenciar claramente que existen empresas que por diversas razones tributarias no poseen impuesto causado, mientras que otras pagan en concepto de impuestos casi todo el beneficio que genera la empresa, aunque en promedio posee un impuesto causado del $28,6 \%$ de los beneficios que generan.

Por otro lado, es importante mencionar que el apalancamiento medio es del 0,697, es decir las empresas ecuatorianas financian en promedio el 69,7\% de sus activos con recursos externos y el 30,3\% restantes con recursos propios. Además, los resultados muestran que ciertas empresas no se encuentran apalancadas con recursos externos.

Respecto al rendimiento sobre los activos, es decir la rentabilidad económica de las empresas ecuatorianas medida como la tasa de devolución del beneficio económico respecto a la inversión en activos totales, esta representa una media del 10,07\% y una mediana del 5,5\%. Mostrando que en promedio los beneficios de las empresas representan el $10 \%$ de los activos totales, mientras que existen empresas cuyos beneficios superan a los activos totales. Por otro lado, se observa que en promedio el $36,38 \%$ y $15,53 \%$ de los activos se encuentran conformados por activos fijos e inventarios respectivamente.

En la Tabla 1 Panel B, se muestran los resultados de la matriz de correlación. Se puede observar que la tasa efectiva tiene una correlación positiva y significativa con el tamaño de las empresas, apalancamiento y con operaciones en el extranjero. Es decir que un aumento en cualquiera de estas variables genera un incremento en la tasa impositiva efectiva y por ende en la carga fiscal. Por otro lado, en la matriz correlación, se puede evidenciar que la tasa efectiva muestra una relación negativa con el rendimiento sobre los activos y con la intensidad del inventario. En el caso de la variable intensidad de capital no se observa una correlación significativa con la tasa impositiva efectiva.

Cabe destacar que la matriz correlación hace un análisis individual de cada variable con la (TIE), sin embargo, si se analiza en su conjunto este comportamiento no necesariamente será significativo, lo cual se podrá observar en la regresión. 


\section{Tabla. 1}

Estadísticas Descriptivas y Matriz de Correlación de Pearson.

\begin{tabular}{|c|c|c|c|c|c|c|c|}
\hline \multicolumn{8}{|c|}{ Panel A: Estadísticas Descriptivas } \\
\hline & $\mathrm{P} 25$ & P50(Mediana) & $\mathrm{P} 75$ & Media & Mínimo & Máximo & \\
\hline Tasa efectiva & 0.22 & 0.241143 & 0.2876064 & 0.2867546 & 0 & 0.9999937 & \\
\hline Tamaño (Ln) & 11.95488 & 12.92918 & 14.00564 & 13.16069 & 10.81981 & 21.23158 & \\
\hline Apalancamiento & 0.3966303 & 0.6599912 & 0.855328 & 0.6073024 & 0 & 1.339948 & \\
\hline Intensidad de Capital & 0.0631953 & 0.2757346 & 0.6325093 & 0.3638066 & 0 & 1 & \\
\hline Intensidad de Inventario & 0 & 0.0242264 & 0.2474922 & 0.1552579 & 0 & 0.9999295 & \\
\hline ROA & 0.0192162 & 0.0550786 & 0.128546 & 0.1007917 & $9.15 \mathrm{E}-10$ & 15.153 & \\
\hline Operaciones en el exterior & 0 & 0 & 0 & 0.0127909 & 0 & 1 & \\
\hline \multicolumn{8}{|c|}{ Panel B: Matriz de correlación } \\
\hline & $\begin{array}{r}\text { Tasa } \\
\text { efectiva }\end{array}$ & Tamaño (Ln) & Apalancamiento & $\begin{array}{c}\text { Intensidad de } \\
\text { Capital } \\
\end{array}$ & $\begin{array}{l}\text { Intensidad de } \\
\text { Inventario }\end{array}$ & ROA & $\begin{array}{c}\text { Operaciones en el } \\
\text { exterior }\end{array}$ \\
\hline Tasa efectiva & 1 & & & & & & \\
\hline Tamaño (Ln) & $0.0852 * * *$ & 1 & & & & & \\
\hline Apalancamiento & $0.1007 * * *$ & $0.0355 * * *$ & 1 & & & & \\
\hline Intensidad de Capital & -0.0032 & $0.0884 * * *$ & $-0.1578 * * *$ & 1 & & & \\
\hline Intensidad de Inventario & $-0.0138 * * *$ & $0.069 * * *$ & $0.2155^{* * *} *$ & $-0.4212 * * *$ & 1 & & \\
\hline ROA & $-0.1736 * * *$ & $-0.0942 * * *$ & $-0.2015 * * *$ & $-0.1879 * * *$ & $-0.1095 * * *$ & 1 & \\
\hline Operaciones en el exterior & $0.0297 * * *$ & $0.2157 * * *$ & $-0.0118 * * *$ & $0.0069 *$ & $-0.0105 * * *$ & $0.0086 * *$ & 1 \\
\hline
\end{tabular}

$* * *$ Significativa al 1\%, ** Significativa al 5\%, *Significativa al $10 \%$

Fuente: elaboración propia 


\section{Efecto de la información contable en la presión fiscal de las empresas ecuatorianas.}

Para determinar el impacto de los factores empresariales en la presión fiscal se procedió a realizar una regresión en panel de datos utilizando los efectos fijos de acuerdo a lo indicado en el test de Hausman. Adicionalmente se realizaron pruebas para observar problemas de heterocedasticidad y se aplicó una prueba de robustez con el fin de corregirlos. Los resultados de la regresión se muestran en la tabla 2.

Dentro de los resultados obtenidos se puede evidenciar que la variable tamaño es negativa y significativa al 1\%, lo cual indica que a mayor tamaño de las empresas menor será la tasa impositiva efectiva de estas, confirmando en este caso la teoría contable del poder político en la economía ecuatoriana. Estos resultados indican que el tamaño si tiene un impacto en la carga fiscal de las empresas por lo cual se acepta la hipótesis 1. Estos datos resultan sorpresivos debido a que, de los 4 años estudiados, tres años y medio estuvieron bajo una reforma total por parte del gobierno que aumentó la presión fiscal respecto al pago del impuesto sobre la renta o sociedades en las grandes empresas, estos resultados sugieren que, a pesar de estos esfuerzos, las grandes compañías incluso tenían en promedio una menor carga fiscal.

Tabla. 2

Factores de la tasa impositiva efectiva/efectos fijos.

\begin{tabular}{lcc}
\hline TIE & Coeficiente & Error estándar \\
\hline Tamaño (Ln) & $\begin{array}{c}-0.017538^{* * *} \\
(-9.59)\end{array}$ & 0.0018288 \\
Apalancamiento & $\begin{array}{c}0.0376557^{* * *} \\
(5.87)\end{array}$ & 0.0064118 \\
Intensidad de Capital & $\begin{array}{c}-0.0031732 \\
(-0.61)\end{array}$ & 0.005212 \\
Intensidad de Inventario & $\begin{array}{c}-0.0034427 \\
(-0.59)\end{array}$ & 0.0058328 \\
ROA & $\begin{array}{c}0.1952787^{* * *} \\
(-7.4)\end{array}$ & 0.026381 \\
Operaciones en el exterior & $\begin{array}{c}0.024025 \\
(24.05)\end{array}$ & 0.0009988 \\
Año 2015 & $\begin{array}{c}0.0134643^{* * *} \\
(10.75)\end{array}$ & 0.0012527 \\
Año 2016 & $0.0235349^{* * *}$ & 0.0015266 \\
Año 2017 & $(15.42)$ & $0.0280783^{* * *}$ \\
$(16.94)$ & 0.0016573 \\
\hline
\end{tabular}

***Significativa al 1\%, ** Significativa al 5\%, *Significativa al 10\%

Fuente: elaboración propia

Respecto a la variable apalancamiento se observa una relación positiva y significativa con la tasa impositiva, es decir mayores niveles de endeudamiento mayor es la carga fiscal de las empresas, aceptando la hipótesis 2. Esta relación positiva puede suceder debido a que dentro de la deuda no solo se incluye a la financiera sino también a la soportada por los proveedores y otros, cuyos intereses no son deducibles de impuestos. Por otro lado, este escenario podría también presentarse debido a la presencia de intereses en exceso del límite deducible en el año fiscal (Fernández et al, 2019). Es necesario recalcar que en Ecuador existen leyes y reglamentos para la deducibilidad de los intereses de deudas contraídas con motivo del giro del negocio, debidamente sustentados, sin exceder los límites de la tasa Esta obra se comparte bajo la licencia Creative Common Atribución-No Comercial 4.0 International (CC BY-NC 4.0) Revista de la Universidad Internacional del Ecuador. URL: https://www.uide.edu.ec/ 
definida por la Junta de Política y Regulación Monetaria y Financiera, y cuyos créditos externos deben encontrarse debidamente registrados en el Banco central del Ecuador. (Ley Orgánica de Régimen Tributario Interno, 2004)

En relación a las variables intensidad de capital e intensidad de inventario, estas son negativas, pero no son significativas por lo cual se puede decir que no existe una relación de causalidad de estas variables con la tasa impositiva efectiva, por ende, no puede considerarse como un factor determinante de la (TIE), rechazando la hipótesis 3.

El coeficiente del ROA es negativo y significativo al 1\%, por lo cual aquellas empresas con mayor rentabilidad sobre sus activos soportan menor carga fiscal que las menos rentables. Estos resultados indican que la carga fiscal afecta la (TIE) de una empresa por lo cual se acepta la hipótesis 4.

Por último, el coeficiente de la variable operaciones en el exterior es positiva, pero no significativa, por lo cual se puede observar que la carga fiscal para empresas con operaciones cien por ciento nacionales es similar a la de empresas con operaciones en el extranjero, rechazando la hipótesis número 5. Por último, se puede observar en la tabla 2 que existen efectos en los diferentes años, dado que los coeficientes de las variables dummies son positivos y significativos.

\section{Conclusiones}

El objetivo principal del presente trabajo es determinar si los distintos factores empresariales, afectan la presión fiscal de las empresas ecuatorianas, para lo cual se utilizó como proxi a la tasa impositiva efectiva. La presión fiscal se la calculó como la ratio entre el impuesto causado y el beneficio antes de impuestos y repartición de utilidades. Entre las variables analizadas como factores empresariales, se encuentra al tamaño, apalancamiento, rentabilidad económica, decisiones de inversión y operaciones en el exterior. Para demostrar esta relación se utilizó como muestra 27877 empresas en el periodo 2014-2017.

Para determinar la relación existente entre la carga fiscal y los factores empresariales, se utilizó un modelo de regresión en datos de panel. Dentro de los resultados más notables se puede mencionar que la variable tamaño si afecta la carga fiscal que soportan las empresas ecuatorianas, dado que a mayor tamaño de la empresa menor es la carga fiscal mostrando de esta forma la teoría del poder político. Lo cual resulta interesante debido a que, en los años de estudios, las grandes empresas estuvieron bajo un mayor escrutinio fiscal por parte del gobierno con la finalidad de que cumplan con sus compromisos tributarios.

Por otro lado, es claro que el apalancamiento de una empresa afecta la carga fiscal de esta, a mayores niveles de endeudamiento, mayor es la presión fiscal de las empresas ecuatorianas. Otra variable importante que se observó fue el rendimiento económico el cual tiene una relación inversa y significativa con la tasa impositiva, por lo cual se evidencia que empresas con mayor rentabilidad sobre sus activos soportan menor carga fiscal que las menos rentables.

Existen ciertas variables estudiadas como la intensidad de capital, intensidad de inventario, y operaciones en el exterior cuyos coeficientes no son significativos bajo el análisis de efectos fijos, por ende, se puede indicar que las decisiones de inversión de una 
empresa y las operaciones que realice en el extranjero no afectan en ningún sentido la presión que estas soportan en el ámbito fiscal.

Finalmente, del estudio realizado se puede concluir que los factores empresariales que afectan la carga impositiva de las empresas ecuatorianas, son el tamaño, apalancamiento y la rentabilidad económica.

\section{Bibliografía}

Adhikari, A., Derashid, C., \& Zhang, H. (2006). Public policy, political connections, and effective tax rates: Longitudinal evidence from Malaysia. Journal of Accounting and Public policy, 25(5), 574-595.

Becker, G. S. (1983). A theory of competition among pressure groups for political influence. The quarterly journal of economics, 98(3), 371-400.

Chiou, Y. C., Hsieh, Y. C., \& Lin, W. (2012). Determinants of effect tax rates for firms listed on China's stock markets: panel models with two-sided censors. The Business \& Management Review, 3(1), 306.

Delgado, F. J., Fernandez-Rodriguez, E., \& Martinez-Arias, A. (2012). Size and other determinants of corporate effective tax rates in US listed companies. International Research Journal of Finance and Economics, 98, 160-165.

Delgado, F. J., Fernández-Rodríguez, E., \& Martínez-Arias, A. (2018). Corporation effective tax rates and company size: evidence from Germany. Economic research-Ekonomska istraživanja, 31(1), 2081-2099.

Fernández, E. (2004). Los factores condicionantes de la presión fiscal empresarial española a partir de la información contable. Especial mención a las decisiones financieras. Spanish Journal of Finance and Accounting/Revista Española de Financiación y Contabilidad, 33(120), 125-159.

Fernández-Rodríguez, E., García-Fernández, R., \& Martínez-Arias, A. (2019). Influence of Ownership Structure on the Determinants of Effective Tax Rates of Spanish Companies. Sustainability, 11(5), 1441.

Fonseca-Díaz, A. R., Fernández-Rodríguez, E., \& Martínez-Arias, A. (2019). Factores empresariales e institucionales condicionantes de la presión fiscal a nivel internacional. Spanish Journal of Finance and Accounting / Revista Española De Financiación Y Contabilidad, 48(2), 224-253.

Irlacher, M., \& Unger, F. (2018). Effective tax rates, endogenous mark-ups and heterogeneous firms. Economics Letters, 173, 51-54.

Lazăr, S. (2014). Determinants of the variability of corporate effective tax rates: Evidence from Romanian listed companies. Emerging Markets Finance and Trade, 50(sup4), 113-131.

Ley Orgánica de Régimen Tributario Interno. Registro Oficial Suplemento de la Republica de Ecuador, 17 de Noviembre de 2004.

Molina, R., \& Barberá, A. (2017). Los determinantes de la presión fiscal empresarial: Evidencia en las empresas de la zona euro durante el período 2004-2014. Harvard Deusto Business Research, 6(1 Especial), 69-82.

Richardson, G., \& Lanis, R. (2007). Determinants of the variability in corporate effective tax rates and tax reform: Evidence from Australia. Journal of accounting and public policy, 26(6), 689-704.

Stickney, C. P., \& McGee, V. E. (1982). Effective corporate tax rates the effect of size, capital intensity, leverage, and other factors. Journal of accounting and public policy, 1(2), 125-152. 
Vintila, G., Gherghina, C., \& Paunescu, R. A. (2018). Study of effective corporate tax rate and its influential factors: Empirical evidence from emerging european markets. Emerging Markets Finance and Trade, 54(3), 571-590.

Watts, R. L., \& Zimmerman, J. L. (1986). Positive accounting theory. Englewood Cliffs, NJ: Prentice Hall.

Zimmerman, J. L. (1983). Taxes and firm size. Journal of accounting and economics, 5, 119149. 